\title{
CARACTERÍSTICAS MORFOLÓGICAS E BIOQUÍMICAS DO PÓLEN COLETADO POR CINCO ESPÉCIES DE MELIPONÍNEOS DA AMAZÔNIA CENTRAL'.
}

\author{
Antonio Carlos MARQUES-SOUZA ${ }^{2}$, Ires Paula de Andrade MIRANDA ${ }^{2}$, Cleonice \\ de Oliveira MOURA ${ }^{2}$, Afonso RABELO ${ }^{2}$, Edelcílio Marques BARBOSA ${ }^{2}$
}

\begin{abstract}
RESUMO - Durante um ano, o pólen transportado por Melipona compressipes manaosensis, Melipona seminigra merrillae, Melipona seminigra seminigra, Frieseomelitta sp. e Scaptotrigona sp. foi coletado diretamente das corbículas das operárias. Feita a identificação dos grãos de pólen constatou-se que a abelha mais generalista foi Scaptotrigona sp., seguida de Frieseomelitta sp., as quais juntas coletaram mais tipos polínicos que as três espécies de meliponas. Duas espécies de plantas: Miconia myrianthera e Myrcia amazonica, tiveram seu pólen coletado pelas cinco espécies de meliponíneos durante o ano todo. Quanto à morfologia do pólen, não houve correlação significativa que comprovasse que as abelhas coletassem o pólen de acordo com a forma, ornamentação e o tamanho dos grãos. As cinco espécies de meliponíneos coletaram pólen de diversas formas e tamanhos. Tudo indica que as abelhas coletam o pólen de acordo com os nutrientes contidos no seu protoplasma. Análises bioquímicas de alguns tipos polínicos encontrados nas corbiculas das abelhas apresentaram alto teor de açúcares solúveis totais, açúcares redutores e amido.
\end{abstract}

Palavras-chave: Amazônia, meliponineos, abelhas sem ferrão, pólen, morfologia polinica

Characteristics of Pollen Collected by Five Species of Meliponid Bees of Central Amazonia.

ABSTRACT - The pollen grains collected by Melipona seminigra merrillae, Melipona
compressipes manaosensis, Melipona seminigra seminigra, Frieseomelitta sp. and Scaptotrigona
sp. were obtained directly from the corbiculae of the worker bees during one year. Identification
of the pollen grains showed that Scaptotrigona sp. was the most generalist bee, followed by
Frieseomelitta sp., which together collected more pollen taxa than the three species of Melipona.
Pollen from three plant species:-Miconia myrianthera, Myrcia amazonica e Tapirira guianensis
- were collected by this five bee species. Regarding pollen morphology, no significant correla-
tions were found that could indicate a preference for certain pollen grain forms, external struc-
tures or sizes of grains. All five meliponids collected pollen of several forms and sizes. It is
indicative that the bees collected pollen in accordance to protoplasmic nutrients. Biochemical
analyses of some pollen types carried on the corbiculae of the bees showed a high concentration
of total soluble sugars, reductive sugars and starch.

Key-words: Amazon, meliponid bees, stingless bees, pollen, pollen morphology

\section{Introdução}

Um dos primeiros passos para o entendimento da biologia de meliponíneos é o conhecimento de seus hábitos alimentares. $\mathrm{O}$ levantamento de plantas fornecedoras tanto de pólen como de néctar, é um estudo importante visando esses elementos essenciais à sobrevivência das abelhas.

O néctar e o pólen são as principais recompensas florais da planta para as abelhas. Quantidade,

\footnotetext{
'Parte do estudo foi financiado pelo CNPq (SWE-Processo n. $200038 / 96-6$ ).

${ }^{2}$ INPA, Coordenação de Pesquisas em Botânica - Caixa Postal 478 - 69083-100, Manaus-AM,

Brasil.E-mail:msouza@inpa.gov.br
} 
concentração e teor de açúcares encontrados no néctar, dão parâmetros para estabelecer o seu nicho trófico e, por isso, são discutidos por vários autores como agentes determinantes de atração alimentar, assim como a distinção que é feita por esses insetos quando da coleta do mesmo (Heinrich \& Raven, 1972; Corbet \& Wilmer, 1981; Southwick et al., 1981; Baker \& Baker, 1983; Gottsberger et al., 1984; Roubik \& Buchmann, 1984; Real \& Rathcke, 1991).

Faltam dados mais concretos para podermos afirmar que as abelhas, de modo semelhante ao néctar, selecionam o pólen através do teor de nutrientes existente no seu protoplasma. Caso fosse assim, não haveria uma gama enorme de tipos polínicos colhidos e não correlacionados entre si e com baixa significância nas amostras (Absy \& Kerr, 1977; Marques-Souza, 1993).

O pólen tem recebido atenção de diversos autores na área da biologia da reprodução (Simpson \& Neef, 1983; Heslop-Harrison \& 1989; Neef \& Simpson, 1993) pelo seu papel nutritivo e como agente de atração dos polinizadores. Dados preliminares atestaram o potencial energético contido no pólen. Petanidou \& Vokou (1990) e Petanidou

(1992) demonstraram a alta energia que possui o pólen quando comparado com outras partes da planta num total de 40 espécies. Colin \& Jones (1980) evidenciaram os valores caloríferos do pólen para as abelhas e outros visitantes florais. Pinheiro et al. (1991) quantificaram as taxas de amido encontradas no pólen de 252 espécies de plantas pertencentes a 63 familias e o tipo de recompensa floral oferecida ao visitante. Essas informações abrem perspectivas para o estudo sobre as preferências específicas de cada abelha por determinada espécie de planta.

Nas observações de MarquesSouza (1993) 50 espécies de plantas pertencentes a 36 gêneros e 21 familias tiveram o pólen coletado por Frieseomelitta varia. Por se tratar de um meliponineo pequeno, nas flores não costumam apresentar comportamento agonistico em relação as outras abelhas. Geralmente abandonam as mesmas quando da chegada das abelhas maiores como as meliponas. Marques-Souza et al. (1995) observaram que operárias de Frieseomelitta varia cediam lugar nas flores para Melipona seminigra merrillae, Melipona rufiventris paraensis, Melipona compressipes manaosensis e para Trigona williana. Segundo os autores isto só não ocorria quando as plantas apresentavam super produção de pólen e podia atender a todos.

Marques-Souza (1999) observou que o maior número de tipos polínicos coletados por parte de Scaptotrigona sp. e Frieseomelitta $\mathrm{sp}$. em relação às meliponas, estava relacionado no fato dessas duas espécies de meliponíneos evitarem a competição pelo mesmo alimento e procuram diversificar as suas coletas para as fontes desprezadas pelas meliponas, o que não foi diferente das observações feitas por Ramalho (1990). 
Com o intuito de colaborar com informações sobre os hábitos de coletas de pólen por meliponíneos na Amazônia, bem como com as preferências alimentares de cada espécie de abelha e o tipo de pólen coletado (tendo como parâmetros, tamanho, forma e o teor de alguns nutrientes do seu protoplasma e se essas características influenciam nas coletas das abelhas), foram coletadas amostras de pólen transportado por cinco espécies de meliponíneos da Amazônia e feitas análises bioquímicas de dez tipos polínicos mais freqüentes nessas amostras.

\section{Material e Métodos}

Os ninhos dos meliponíneos foram introduzidos no Campus do INPAInstituto Nacional de Pesquisas da Amazônia, localizado nos arredores de Manaus, com coordenadas geográficas $3^{\circ} 08^{\prime} \mathrm{S}$ e $60^{\circ} 10^{\prime} \mathrm{W}$ a uma altitude de 40,33 metros.

As amostras de pólen foram obtidas de cinco espécies de meliponíneos da Amazônia: Frieseomelitta sp., Melipona compressipes manaosensis (Schwarz, 1932), Melipona seminigra merrillae (Cockerell, 1919), Melipona seminigra seminigra (Friese, 1903) e Scaptotrigona sp.

O período estabelécido para obtenção das amostras de pólen foi de um ano, com início em agosto de 1995 e término em julho de 1996 . O horário das coletas dos pólen corbiculares foi sempre entre 07:00 e 09:00 hs.

As amostras de pólen foram obtidas de cinco abelhas de cada espécie, capturadas aleatoriamente quando do fechamento da entrada das colméias. As bolotas de pólen foram retiradas das corbículas com o auxílio de um estilete e acondicionadas em vidros esterilizados. As abelhas foram libertadas logo após a retirada de seus carregamentos.

Para a análise bioquímica, em determinados dias de cada mês, foi colhido pólen de bolotas de cores iguais para cada espécie de abelha e acondicionados separadamente em tubos. Em seguida as bolotas de pólen foram diluídas em $10 \mathrm{ml}$ de água destilada, com o auxílio de um misturador marca Vortex-Genie, e centrifugados a $1.500 \mathrm{rpm}$. Repetiu-se o processo de lavagem e de separação dos grãos para a retirada das secreções salivares e de néctar que são utilizados pelas abelhas para agregar o pólen em bolotas. Depois de lavados os grãos de pólen foram colocados em placas de vidro, onde permaneceram durante 48 horas em sílica gel para total secagem. Feita a identificação dos grãos, bolotas de pólen pertencentes a uma mesma espécie de planta foram juntadas e pesadas em uma balança analítica modelo Mettler PC 2000. Cada amostra foi dividida em três sub amostras, com pesos que variaram de 0,10 a 0,15 gramas.

Para a leitura das absorbâncias de açúcares solúveis totais do pólen, para cada subamostra foram feitas duas curvas padrões, uma com xilose a $0,01 \%$ nas concentrações de $10 \mu \mathrm{g}$ à $90 \mu \mathrm{g}$, lidas a $480 \mathrm{~nm}$ para cálculo de pentose e outra com glicose a 
$0,01 \%$, utilizando as mesmas concentrações, lidas a $490 \mathrm{~nm}$, para cálculo das hexoses.

Para a leitura das absorbâncias dos açúcares redutores, a curva padrão foi determinada com uma solução de glicose a $0,01 \%$, com concentrações variando de $10 \mu \mathrm{g}$ a $90 \mu \mathrm{g}$, lidas a 540 $\mathrm{nm}$ em um espectrofotômetro MICRONAL B 380.

$\mathrm{Na}$ hidrólise do amido, as absorbâncias foram lidas a $490 \mathrm{~nm}$ e os valores obtidos multiplicados pelo fator de correção 0,9 para a conversão em quantidades de amido. Para a realização da curva padrão, foi utilizada uma solução de glicose a $0,01 \%$, cujas concentrações variaram de 5 a $45 \mu \mathrm{g}$.

Como efeito comparativo da análise bioquímica do protoplasma dos grãos de pólen, utilizou-se como material de referência o pólen das palmeiras Elaeis oleifera e Elaeis guineensis.

Quanto à identificação dos tipos polínicos, de cada amostra das bolotas foi retirado uma porção pequena que foi acetolisada pelo método de Erdtman (1960). Em seguida as amostras de pólen foram montadas em lâminas com gelatina glicerinada e lutadas com parafina. Foram preparadas duas lâminas de pólen para cada amostra e feitas as medidas dos eixos polar e equatorial em 25 diferentes grãos de uma mesma espécie, utilizando uma ocular micrométrica. Realizadas as medidas dos eixos polar e equatorial, foram feitos os cálculos estatísticos das médias, desvio padrão da média e intervalo de confiança. Para as espessuras da sexina e da nexina fo- ram calculadas somente as médias aritméticas.

\section{Resultados e Discussão}

\section{Diversidade polínica}

A quantidade de espécies vegetais que tiveram o seu pólen coletado pelas cinco espécies de meliponíneos no periodo de um ano está sumariado na Tabela 1. Os dados mostram que a abelha Scaptotrigona sp. coletou maior número de tipos polínicos que os outros meliponíneos em outubro, novembro, janeiro, fevereiro e março, além de coletar igual número que Melipona seminigra merrillae e Melipona seminigra seminigra no mês de julho. A mais ampla coleta por Scaptotrigona sp. foi feita no mês de janeiro, compreendendo 37 táxons pertencentes a 33 gêneros e 22 famílias.

Em seguida destacou-se Melipona seminigra seminigra que coletou mais tipos polínicos que as outras abelhas em abril e maio. Melipona seminigra merrillae coletou mais que os outros meliponíneos em junho, além de igual número que Frieseomelitta sp. em setembro. A abelha Melipona compressipes manaosensis coletou mais tipos polínicos em agosto. Frieseomelitta sp. coletou mais tipos polinicos que as demais em dezembro. A atividade de coletas destas abelhas corresponde a observações feitas por Marques-Souza (1993; 1995; 1999).

Houve repetições na coleta de tipos polínicos durante vários meses. Espécies como Cassia grandis (Caesalpiniaceae) forneceram pólen 
Tabela 1. Distribuição mensal quantitativa dos tipos polinicos coletados pelas cinco espécies de meliponíneos.

\begin{tabular}{|c|c|c|c|c|c|c|c|c|c|c|c|c|c|c|c|c|}
\hline & & & Fs & & & $\mathrm{Mcm}$ & & & Msm & & & Mss & & & Ss & \\
\hline A & $M$ & & & & & & & & & & & & & & & \\
\hline$N$ & $\dot{E}$ & N.o de & Dist. & Dist. & N.ode & Dist. & Dist. & N.ode & Dist. & Dist. & N.o de & Dist. & Dist. & N.o de & Dist. & Dist. \\
\hline \multirow[t]{3}{*}{0} & $S$ & espécies & por & por & espécies & por & por & espécies & por & por & espécies & por & por & espécies & por & por \\
\hline & & coletadas & gêneros & familias & coletadas & gêneros & fam ilias & coletadas & gêneros & familias & coletadas & gêneros & fam ilias & coletadas & gêneros & familias \\
\hline & Agosto & 14 & 11 & 9 & 23 & 18 & 11 & 15 & 15 & 13 & 21 & 20 & 10 & 18 & 18 & 13 \\
\hline 1 & Setem bro & 20 & 18 & 16 & 14 & 13 & 11 & 20 & 20 & 15 & 17 & 16 & 12 & 19 & 19 & 13 \\
\hline 9 & Outubro & 17 & 15 & 15 & 20 & 15 & 12 & 19 & 15 & 12 & 27 & 25 & 17 & 28 & 25 & 18 \\
\hline 9 & Novem bro & 13 & 11 & 11 & 13 & 11 & 9 & 12 & 11 & 9 & 12 & 11 & 10 & 25 & 17 & 12 \\
\hline \multirow[t]{2}{*}{5} & Dezem bro & 19 & 17 & 16 & 11 & 8 & 6 & 9 & 8 & 7 & 12 & 12 & 10 & 17 & 12 & 12 \\
\hline & Janeiro & 11 & 10 & 8 & 12 & 11 & 10 & 14 & 12 & 10 & 9 & 8 & 7 & 37 & 33 & 22 \\
\hline 1 & Fevereiro & 14 & 13 & 11 & 14 & 12 & 11 & 14 & 14 & 10 & 19 & 15 & 13 & 21 & 19 & 17 \\
\hline 9 & Março & 14 & 13 & 12 & 12 & 9 & 8 & 11 & 11 & 10 & 11 & 11 & 7 & 21 & 19 & 15 \\
\hline 9 & Abril & 13 & 12 & 11 & 12 & 9 & 6 & 16 & 16 & 12 & 18 & 14 & 12 & 14 & 14 & 12 \\
\hline \multirow[t]{3}{*}{6} & Maio & 10 & 10 & 10 & 8 & 6 & 4 & 17 & 16 & 12 & 20 & 18 & 11 & 15 & 14 & 12 \\
\hline & Junho & 7 & 6 & 6 & 8 & 7 & 7 & 22 & 20 & 15 & 20 & 17 & 12 & 11 & 11 & 8 \\
\hline & Julho & 11 & 11 & 11 & 12 & 7 & 7 & 16 & 14 & 10 & 16 & 15 & 12 & 16 & 16 & 13 \\
\hline
\end{tabular}

Obs.: Fs=Frieseomelitta sp.; Mcm=Melipona compressipes manaosensis; $M s m=$ Melipona seminigra merrillae;

Mss=Melipona seminigra seminigra; $\mathrm{Ss}=$ Scaptotrigona $\mathrm{sp}$. 
para Melipona compressipes manaosensis durante dez meses consecutivos. Outras, como Leucaena sp. (Mimosaceae) forneceram pólen para Melipona seminigra seminigra durante os doze meses do ano. As espécies Stryphnodendron guianense (Mimosaceae) e Matayba sp. (Sapindaceae) tiveram seu pólen coletado por Scaptotrigona sp. em onze meses, enquanto as espécies Miconia myrianthera (Melastomataceae) e Myrcia amazonica (Myrtaceae) tiveram seu pólen coletado pelas cinco espécies de meliponineos durante $o$ ano todo.

A espécie Morus sp. (Moraceae) foi explorada somente por Frieseomelitta sp., assim como o táxon Portulacaceae tipo cuja coleta de pólen se restringiu a Scaptotrigona sp. e um pouco a Melipona compressipes manaosensis. $\mathrm{O}$ pólen de Zanthoxylum sp. (Rutaceae), foi intensamente coletado por Melipona seminigra seminigra em agosto. Outras espécies como Stryphnodendron guianense (Mimosaceae), Tapirira guianensis, Spondias mombin (Anacardiaceae) e Physocalymma scaberrimum (Lythraceae) tiveram seu pólen coletado em dias e meses intercalados por todos os meliponíneos. Já as espécies Aparisthmium cordatum (Euphorbiaceae), Bellucia grossularioides (Melastomataceae) e Genipa americana (Rubiaceae) foram visitadas para a coleta de pólen por quatro tipos de abelhas em oito meses intercalados, enquanto Thyrsodium sp. (Anacardiaceae) foi visitada por três tipos de meliponíneos em quatro meses diferentes. As espécies Cassia grandis (Caesalpiniaceae) e Psidium sp. (Myrtaceae) tiveram seus pólen coletados por dois tipos de meliponíneos em três meses distintos (Tab. 2).

Em estudos de plantas fornecedoras de pólen e/ou néctar, há cada vez mais a constatação de que muitas plantas são utilizadas por todas as espécies de abelhas, mas há aquelas plantas isoladas que só determinada espécie de abelha visita. É natural que dentro da comunidade em estudo somente algumas destas plantas sejam mais procuradas por uma espécie de abelha. São essas preferências alimentares de cada abelha, somado

Tabela 2. Distribuição qualitativa dos 18 tipos polinicos mais significativos coletados por cinco espécies de meliponíneos.

\begin{tabular}{|c|c|c|c|c|c|c|c|c|c|c|c|c|c|c|c|c|c|c|}
\hline \multirow[b]{2}{*}{$\begin{array}{l}\text { Espécies } \\
\text { de } \\
\text { abelhas }\end{array}$} & \multicolumn{18}{|c|}{ Táxons vegetais } \\
\hline & 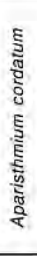 & 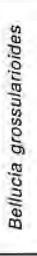 & 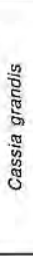 & 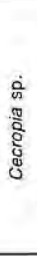 & 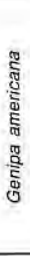 & 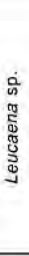 & 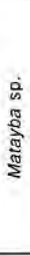 & 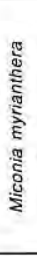 & $\begin{array}{l}\frac{0}{0} \\
\text { yू } \\
\frac{0}{2}\end{array}$ & 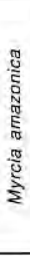 & 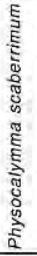 & 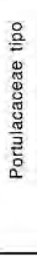 & 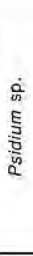 & 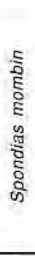 & 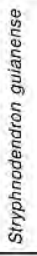 & 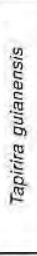 & $\begin{array}{l}\text { के } \\
\text { है } \\
\text { है } \\
\text { o } \\
\text { जू } \\
\text { है }\end{array}$ & 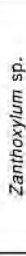 \\
\hline Frieseomelitta sp. & $x$ & & & $\mathrm{x}$ & $x$ & $x$ & $x$ & $x$ & $x$ & $x$ & $x$ & & & $x$ & $x$ & $x$ & $x$ & \\
\hline Melipona compressipes manaosensis & & $x$ & $x$ & $\mathrm{x}$ & $x$ & $x$ & $\mathrm{x}$ & $\mathrm{x}$ & & $x$ & $x$ & $\mathrm{x}$ & $\mathrm{x}$ & $x$ & $x$ & $x$ & $x$ & \\
\hline Melipona seminigra merrillae & $x$ & $\mathrm{x}$ & $x$ & $x$ & & $x$ & $\mathrm{x}$ & $x$ & & $\mathrm{x}$ & $\mathrm{x}$ & & & $x$ & $x$ & $x$ & & \\
\hline Melipona seminigra seminigra & $x$ & $x$ & & $x$ & $\mathrm{x}$ & $\mathrm{x}$ & $x$ & $x$ & & $x$ & $x$ & & & $x$ & $x$ & $\mathrm{x}$ & & $x$ \\
\hline Scaptotrigona sp. & $x$ & $x$ & & $x$ & $\mathrm{x}$ & $x$ & $x$ & $x$ & & $x$ & $x$ & $x$ & $x$ & $x$ & $x$ & $x$ & $x$ & \\
\hline
\end{tabular}


com a monopolização das fontes mais atrativas por um determinado visitante, que torna o nicho ecológico de uma espécie diferente da outra (Case \& Gilpin, 1974; Absy et al., 1980). Por este motivo as espécies Miconia myrianthera, Myrcia amazonica, Stryphnodendron guianense, entre outras, foram exploradas por todos os meliponíneos durante o ano todo, enquanto outras espécies como Morus sp. e Zanthoxylum sp. foram visitadas somente por uma determinada espécie de meliponíneo. Estas plantas e outras fazem parte das espécies "maiores" citadas por Heinrich (1979), onde apenas uma pequena parcela da população floral fornece a maior quantidade do alimento para as abelhas.

A dispersão na procura de fontes alternativas de alimento pode ser um problema de comunicação entre as campeiras com as demais abelhas da colméia, não conseguindo passar para elas a localização correta das fontes em potencial. No caso, é fato que durante o período estudado, Frieseomelitta sp. e Scaptotrigona sp. diversificaram suas coletas mais do que as três espécies de meliponas e, conseqüentemente, coletaram o pólen de um número maior de espécies de plantas (Tab. 1).

Tudo indica que a comunicação tenha sido o diferencial nas coletas dos meliponíneos. As três espécies de meliponas conseguiram repassar a localização exata das fontes mais produtivas, o que pode não ter acorrido com Frieseomelitta sp. e Scaptotrigona sp. que se dispersaram na procura de outras fontes. Portanto, as três espécies de meliponas restringiram as suas coletas a um número menor de plantas. Outra explicação deste fato poderia ser uma maior dispersão causada a Frieseomelitta sp. e Scaptotrigona sp. pelas três espécies mais vigorosas de meliponas, conforme assinalado por Marques-Souza (1999).

\section{Morfologia polínica}

Com relação à morfologia polínica, os resultados mostram que 18 espécies de plantas foram as mais freqüentes nas coletas dos meliponíneos durante vários meses. Os dados morfométricos estão apresentados na Tabela 3 .

Alguns fatores relacionados com as características do pólen são discutidos por alguns autores como influenciadores na coleta de pólen pelas abelhas. Entre as características envolvidas nesta discussão estão tamanho dos grãos de pólen, rugosidade, aderência, qualidade, disponibilidade, umidade, peso, pulverulência, odor, cor, teor de azoto, etc. (Pla Dalmau, 1961 citado por Cortopassi-Laurino, 1982).

Sobre o tamanho dos grãos de pólen como um dos determinantes na coleta das abelhas, Silveira (1991) constatou que grãos de pólen grandes, como o de Mabea sp. (Euphorbiaceae) e de espécies de Sterculiaceae, apresentaram uma contribuição volumétrica maior do que os de $\mathrm{Ce}$ cropia sp. e de espécies de Myrtaceae nas bolotas transportadas por abelhas. Cortopassi-Laurino \& Ramalho (1988) observaram que as Apis mellifera não coletaram grãos de pólen com tamanho 
Tabela 3. Dados morfométricos e ornamentação dos grãos de pólen de 18 taxa coletados pelas abelhas.

\begin{tabular}{|c|c|c|c|c|c|c|c|c|c|c|}
\hline \multirow{2}{*}{$\begin{array}{c}\text { Espécie de } \\
\text { Planta }\end{array}$} & \multirow{2}{*}{$\begin{array}{l}\text { Abelha } \\
\text { coletora }\end{array}$} & \multirow{2}{*}{$\begin{array}{l}\text { Tamanho } \\
\text { dos grấos }\end{array}$} & \multirow{2}{*}{$\begin{array}{c}\text { Tipo da } \\
\text { Ornamentação }\end{array}$} & \multirow[t]{2}{*}{ Forma } & \multirow[t]{2}{*}{ Ámbito } & \multicolumn{2}{|c|}{ Intervalo de Confiança } & \multicolumn{2}{|c|}{ Exina } & \multirow{2}{*}{$\begin{array}{c}\text { Relaçăo } \\
\text { P/E }\end{array}$} \\
\hline & & & & & & E. P. & E. Eq. & Sexina & Nexina & \\
\hline Aparisthmium cordatum & Msm & Médio & Reticulada & Esferoidal & Circular & $34,25 \pm 0,57(31,3-39,9)$ & $35,62 \pm 0,46(31,3-39,9)$ & 1,4 & 1.4 & 1,0 \\
\hline Bellucia grossularioides & Msm & Pequeno & Reticulada & Prolato & Circular & $17,61 \pm 0.30(14,2-19,9)$ & $12,6 \pm 0,14(8,5 \cdot 14,2)$ & 0,7 & 0,6 & 1,4 \\
\hline Cassia grandis & Mcm & Grande & Reticulada & Prolato esteroidal & Circular & $61,78 \pm 0,19(57,0-71,2)$ & $48,27 \pm 0,42(42,7-54,1)$ & 1,3 & 1,3 & 1,3 \\
\hline Genipa americana & $\mathrm{Mcm}$ & Médio & Reticulada & Oblato & Circular & $30,89 \pm 0,80(25,6-42,7)$ & $26,26 \pm 0,69(22,8-37,0)$ & 0,6 & 0,6 & 1,4 \\
\hline Leucaena sp. & Msm & Grande & Reticulada & Prolato esteroidal & Circular & $61,21 \pm 0,65(48,4-68,4)$ & $46,45 \pm 0,45(39,9-51,3)$ & 1,6 & 1,7 & 1.1 \\
\hline Miconia myrianthera & Mss & Pequeno & Psilada & Prolato esteroidal & Circular & $24,90 \pm 0,89(19,9-28,5)$ & $19,66 \pm 0,40(14,2-25,6)$ & 0.9 & 0.6 & 1,3 \\
\hline Morus sp. & Fs & Pequeno & Reticulada & Prolato esteroidal & Circular & $14,68 \pm 0,35(14,2-17,1)$ & $10,65 \pm 0,26(11,4-14,2)$ & 0,7 & 0,5 & 1.4 \\
\hline Physocalymma scaberrimum & Msm & Médio & Reticulada & Oblato esferoidal & Circular & $25,51 \pm 0,22(28,5-34,2)$ & $26,67 \pm 0,30(22,8 \cdot 31,3)$ & 0.9 & 0,7 & 0,9 \\
\hline Portulacaceae tipo & Ss & Grande & Reticulada & Prolato & Circular & $58,93 \pm 0,60(51,3-68,4)$ & $32,83 \pm 0,20(31,3-42,7)$ & 1,2 & 1.2 & 1,8 \\
\hline Spondias mombin & Msm & Grande & Estriada & Prolato & Circular & $54,43 \pm 0,50(48,4-59,8)$ & $37.79 \pm 0,41(31,3 \cdot 42,7)$ & 1,2 & 0.9 & 1,4 \\
\hline Tapirira guianensis & Ss & Médio & Estriada & Oblato esferoidal & Circular & $23,09 \pm 0,34(28,5-37,0)$ & $25,02 \pm 0,39(22,8-28,5)$ & 1,0 & 0.6 & 0.9 \\
\hline Thyrsodium sp & Ss & Médio & Estriada & Prolato esferoidal & Circular & $32,54 \pm 0,28(28,5-37,0)$ & $23,76 \pm 0,70(19,9-25,6)$ & 1,0 & 0,9 & 1,4 \\
\hline \multirow[t]{2}{*}{ Zanthoxylum sp. } & Mss & Médio & Rugosa & Prolato esteroidal & Circular & $26,61 \pm 0,21(22,8-28,5)$ & $20,91 \pm 0,21(17,1-22,8)$ & 1,0 & 0.7 & 1.3 \\
\hline & & & & & & DM & $\mathrm{Dm}$ & & & $\mathrm{DM} / \mathrm{Dm}$ \\
\hline Cecropia sp. & Fs & Pequeno & Reticulada & Prolato sub esteroidal & Sub circular & $12,25 \pm 0,40(11,4-14,2)$ & $8,15 \pm 0,38(5,7 \cdot 8,5)$ & 0,6 & 0.5 & 1,5 \\
\hline Matayba sp. & Ss & Médio & Reticulada & Prolato esteroidal & Circular & $29,64 \pm 0,20(25,6-39,9)$ & $17,7 \pm 0,32(14,2-22,8)$ & 0.9 & 0,6 & 1,7 \\
\hline Myrcia amazonica & Mss & Pequeno & Psilada & Prolato & Triangular & $22,62 \pm 2,10(19,9-25,6)$ & $15,24 \pm 0.27(11,4-17,1)$ & 0,7 & 0.6 & 1,5 \\
\hline Psidium sp. & $\mathrm{Mcm}$ & Pequeno & Psilada & Oblato esferoldal & Triangular & $18,18 \pm 0,40(17,1-19,9)$ & $17,80 \pm 0,26(14,2-19,9)$ & 0,6 & 0,6 & 1,0 \\
\hline Stryphnodendron guianense & Ss & Pequeno & Psilada & Oblato esferoidal & Circular & $23,31 \pm 0.49(19,9-31,3)$ & $16,01 \pm 0,40(14,2-19,9)$ & 0.7 & 0,5 & 1,4 \\
\hline
\end{tabular}

\section{\begin{tabular}{ll}
\hline E. P. $=$ Eixo Polar & $\mathrm{DM}=$ Diâmetro maior \\
E. Eq. $=$ Eixo Equatorial & $\mathrm{Dm}=$ Diâmetro menor
\end{tabular}}

Msm=Melipona seminigra merrillae

Mcm=Melipona compressipes manaosensis

Mss=Melipona seminigra seminigra

Ss=Scaptotrigona sp.

$\mathrm{Fs}=$ Frieseomelitta sp. 
superior a $71 \mathrm{~mm}$, enquanto que a Trigona spinipes coletaram pólen com tamanho entre $20-30 \mathrm{~mm}$, porém estenderam as suas coletas para grãos de pólen de tamanho grande. Santos (1991) constatou que as operárias de Melipona seminigra merrillae coletaram grãos de pólen de todos os tamanhos e com isso não estabeleceram uma relação de preferência. Verificou-se no presente estudo que entre 18 tipos polínicos coletados pelas cinco espécies de meliponíneos há grãos de pólen de tamanhos pequeno, médio e grande, não havendo um que se destacasse mais que o outro (Tab. 2 e 3). Quanto à superfície do pólen, a maioria apresentou exina reticulada, alguns psilada (lisa) e estriada. Apenas um tipo polínico, Zanthoxylum sp., apresentou exina levemente rugosa .

Pelos dados obtidos é possivel afirmar que o tamanho do pólen e a estrutura da exina não tiveram um papel preponderante nas coletas efetuadas pelos cinco tipos de meliponíneos aqui estudados, pois as espécies mais exploradas Myrcia amazonica e Miconia myrianthera possuem grãos de pólen pequenos e de superfície lisa, enquanto que os de Leucaena sp. e Cassia grandis possuem pólen grande e de superfície reticulada e os de Tapirira guianensis possuem tamanho médio e exina estriada (Fig. 1).

Há outros fatores interagindo sobre as coletas. Grãos de pólen grandes (como os de Leucaena sp. e Cassia grandis) têm uma contribuição volumétrica maior quando agregados em bolotas que os grãos de pólen médios (Aparisthmium cordatum e Tapirira guianensis) e pequenos (Myrcia amazonica e Miconia myrianthera), ou seja, uma menor quantidade de grãos de pólen grandes é suficiente para superar um número bem maior de grãos médios/pequenos. Provavelmente, o tempo gasto na coleta pelas abelhas e a permanência nas flores, deve ser menor com os grãos grandes e maior com os grãos pequenos, uma vez que elas precisam coletar uma quantidade mais elevada de grãos pequenos para formar uma massa polínica, que com menos tempo pode ser conseguida com grãos grandes (Marques-Souza, 1993; 1996).

\section{Nutrientes do pólen}

A análise do teor de alguns nutrientes encontrados no protoplasma de dez tipos polínicos que em determinado dia foram os mais freqüentes nas coletas dos meliponíneos, mostrou que as taxas de xilose e glicose do pólen de Cassia grandis, Myrcia amazonica, Bellucia grossulariodes e Psidium sp. ficaram na faixa dos 200 $\mathrm{mg} / \mathrm{g}$ de matéria fresca, o pólen de Aparisthmium cordatum, Morus sp., Thyrsodium sp., Miconia myrianthera, Zanthoxylum sp. e o táxon Portulacaceae tipo na faixa dos $300 \mathrm{mg} /$ $\mathrm{g}$ de matéria fresca.

Quanto ao teor de açúcares redutores, os dados mostraram que o pólen de Cassia grandis e Myrcia amazonica apresentou 443,08 e $456,55 \mathrm{mg} / \mathrm{g}$ de matéria fresca, respectivamente. Quanto ao teor de amido, o pólen de Aparisthmium 


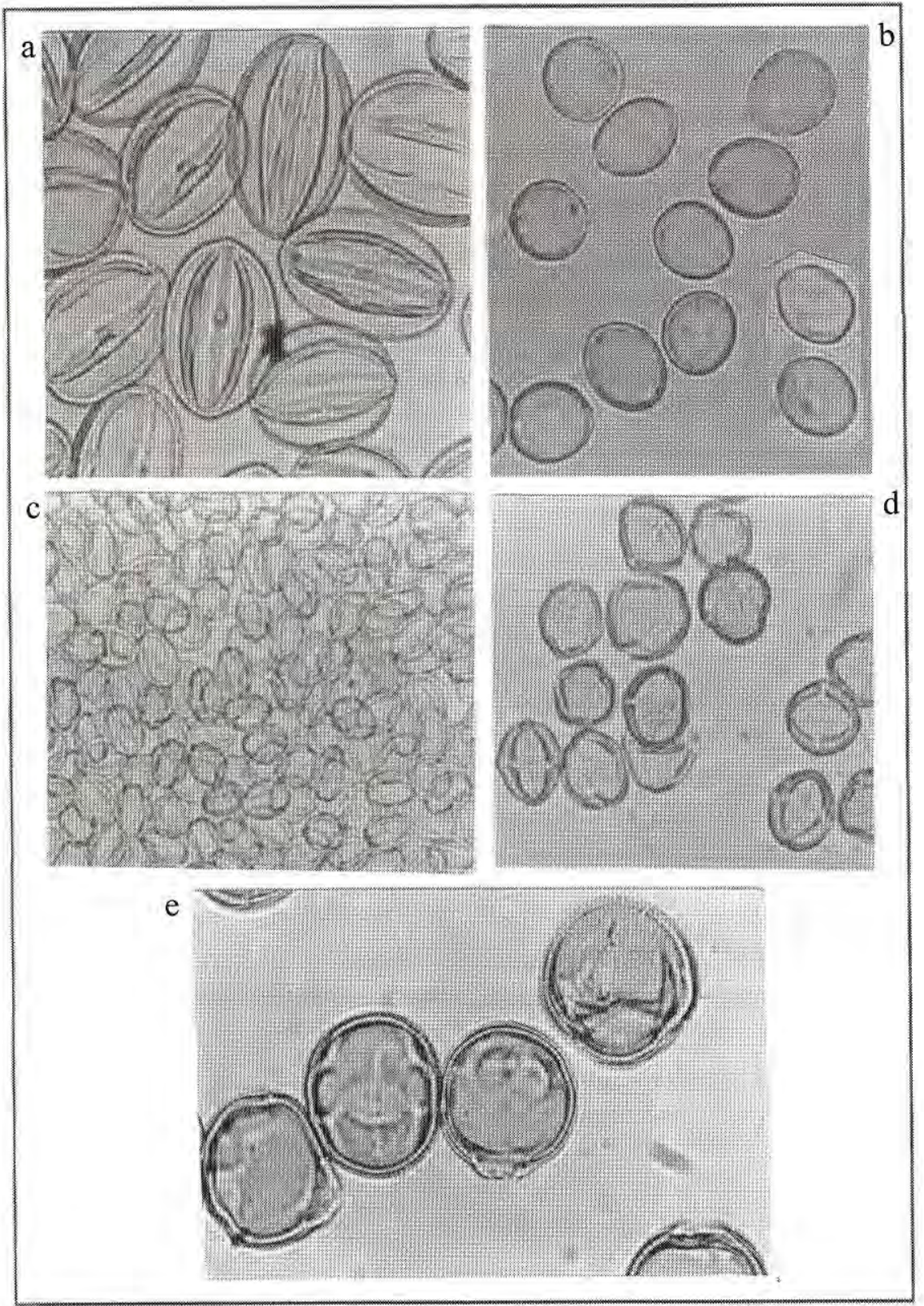

Figura 1.-Grãos de pólen transportados pelas abelhas. a) Cassia grandis (400x). b) Morus sp. (1000x), c) Miconia myrianthera (400x), d) Tapirira guianensis (400x). e) Byrsonima sp. (1000x). 
cordatum apresentou o maior valor com $8,77 \mathrm{mg} / \mathrm{g}$ de matéria fresca, enquanto o de Myrcia amazonica apresentou o menor valor com $0,07 \mathrm{mg} /$ $\mathrm{g}$ de matéria fresca (Tab. 4).

Do ponto de vista do teor de nutrientes do pólen, poucos são os dados que comprovam que os grãos de pólen grandes contêm todas as substâncias que as abelhas necessitam. Os grãos de pólen grandes de Cassia grandis foram os que apresentaram uma das menores taxas de xilose, glicose e de açúcares redutores; não diferiu muito de grãos de pólen pequenos como o de Bellucia grossularioides, Myrcia amazonica e Miconia sp.

No ítem amido, os grãos de pólen médios de Aparisthmium cordatum se destacaram dos demais por apresentar a maior taxa com 8,77 de $\mathrm{mg} / \mathrm{g}$ matéria fresca, enquanto os grãos de pólen grandes do táxon Portulacaceae tipo e Cassia grandis apresentaram taxas bem menores, respectivamente 0,80 e $0,49 \mathrm{de} \mathrm{mg} / \mathrm{g}$ matéria fresca. Dependendo da espécie, a síntese do amido acontece tanto no pólen imaturo, que ainda está dentro dos botões como no mais desenvolvido. Porém, em todos os deis casos, o amido permanece no pólen até a sua dispersão (Pinheiro et al., 1991).

Pelo que se pôde constatar que, assim como as abelhas conseguem selecionar as espécies vegetais pela composição química e a concentração de açúcares de seu néctar (Louveaux, 1968), o mesmo acontece com o pólen, cujo valor nutritivo as abelhas conseguem diferenciar. Mesmo assim, se faz necessário observar outros fatores que interagem sobre as coletas

Tabela 4. Médias dos teores de carboidratos ( $\mathrm{mg} / \mathrm{g}$ matéria fresca) encontrados no pólen coletado por quatro espécies de meliponíneos.

\begin{tabular}{clcccc}
\hline Abelha & \multicolumn{1}{c}{ Planta } & Xilose & Glicose & Aç. Red. & Amido \\
\hline$\ldots$ & Elaeis oleifera Cortes & 113,44 & 125,03 & 32,07 & 6,46 \\
$\ldots$ & Elaeis guineensis Jacq & 150,25 & 173,48 & 14,64 & 2,34 \\
Msm & Aparisthmium cordatum(Juss.) Baill & 311,79 & 304,00 & 608,39 & 8,77 \\
Msm & Bellucia grossularioides Triana & 248,55 & 251,01 & 530,49 & 0,86 \\
Mcm & Cassia grandis Linn. & 226,65 & 232,57 & 443,08 & 0,49 \\
Mss & Lindackeria paludosa Gilg & 365,90 & 411,29 & 789,84 & 2,29 \\
Mss & Miconia myrianthera Benth. in Hook & 359,08 & 347,76 & 618,39 & 3,87 \\
Mcm & Miconia sp. & 260,99 & 272,23 & 559,45 & 0,26 \\
Mss & Myrcia amazonica DC. & 232,20 & 245,17 & 456,55 & 0,07 \\
Ss & Portulacaceae tipo & 335,72 & 328,08 & 601,60 & 0,80 \\
Ss & Thyrsodium sp. & 333,23 & 365,49 & 757,06 & 3,54 \\
Msm & Vataireopsis speciosa Ducke & 317,64 & 340,08 & 722,13 & 1,56 \\
\hline
\end{tabular}

Obs.: Msm=Melipona seminigra merrillae

$\mathrm{Mcm}=$ Melipona compressipes manaosensis

Mss=Melipona seminigra seminigra

$\mathrm{Ss}=$ Scaptotrigona $\mathrm{sp}$.

Aç. Red.=Açúcar redutor 
feitas pelas abelhas, entre eles a diminuição sazonal do alimento, morfologia das peças florais que podem facilitar ou dificultar as coletas, a antese das flores, oferta de pólen/néctar nas flores que estão presentes somente em certos períodos do ano, o número de insetos competidores, a distância das fontes mais atrativas, comunicação entre as abelhas, as pragas e os inimigos naturais, entre outros.

\section{Bibliografia citada}

Absy, M.L.; Kerr, W.E. 1977. Algumas plantas visitadas para a obtenção de pólen por operárias de Melipona seminigra merrillae em Manaus. Acta Amazonica, 7(3): 309315.

Absy, M.L.; Bezerra, E.B.; Kerr, W.E. 1980. Espécies nectariferas utilizadas por duas espécies de Melipona da Amazônia. Acta Amazonica, 10(2): 271-281.

Baker, H.G.; Baker, I. 1983. Floral nectar sugar constituintes in relation to pollination type. In: Jones, C.E.; Little, R.J. (Eds). Handbook of experimental pollination biology. Van Nostrand-Reinhold, New York, USA. p.117-141.

Case T.J.; Gilpin, M.E. 1974. Interference competition and niche theory. Proc. Nat. Acad. Sci. USA, 71(8): 3073-3077.

Colin, L.J.; Jones, C.E. 1980. Pollen energetics and pollination modes. Amer. J. Bot., 67: 210-215.

Corbet, S.A.; Wilmer, P.G. 1981. The nectar of Justicia and Columnea: composition and concentracion in a humid tropical climate. Oecologia, 51: 412-418.

Cortopassi-Laurino, M. 1982. Divisão dos recursos tróficos entre abelhas sociais principalmente em Apis mellifera Linné e Trigona (Trigona) spinipes Fabricius (Apinae, Hymenoptera). Tese de Doutorado, Instituto de Biociências, USP, São Paulo (SP), 180p.

Cortopassi-Laurino, M.; Ramalho, M. 1988.
Pollen harvest by africanized Apis mellifera and Trigona spinipes in São Paulo botani$\mathrm{cal}$ and ecological views. Apidologie, 19(1): $1-24$.

Erdtman, G. 1960. The acetolisys method in a revised description. Sv. Bot. Tidskr. Lund., 54(4): 561-564.

Gottsberger, G.; Schrauwen, J.; Liskens, H.F. 1984. Amino acids and sugars in nectar, and their putative evolutionary significance. Pl. Syst. Evol., 145: 55-77.

Heinrich, B.; Raven, P. 1972. Energetics and pollination ecology. Science, 176: 597-602.

Heinrich, B. 1979. "Majoring" and "minoring" by foraging bumblebees, Bombus vagans: an experimental analysis. Ecology, 60(21): 245-255.

Heslop-Harrison, J.; Heslop-Harrison, Y. 1989. Conformation and movement of the vegetative nucleus of the angiosperm pollen tube: association with the actin cytoskeleton. J. Cell. Sci., 93: 299-308.

Louveaux, J. 1968. L'analyse pollinique des miels, In: Chauvin, R. (Ed). Traité de biologie de l'abeille. Vol. 3. Masson et Cie. Paris, France. p.325-362.

Marques-Souza, A.C. 1993. Espécies de plantas visitadas para a coleta de pólen por cinco tipos de meliponineos da Amazônia. Dissertação de Mestrado, Instituto Nacional de Pesquisas da Amazônia/Fundação Universidade do Amazonas. Manaus, Amazonas. 114p.

Marques-Souza, A.C.; Absy, M.L.; Kerr, W.E.; Peralta, F.J.A 1995. Pólen coletado por duas espécies de meliponineos ( $\mathrm{Hy}-$ menoptera: Apidae) da Amazônia. Rev. Brasil. Biol., 55(4): 855-864.

Marques-Souza, A.C. 1996. Fontes de pólen exploradas por Melipona compressipes manaosensis (Apidae: Meliponinae), abelha da Amazonia Central. Acta Amazonica, 26(1/2): 77-86.

Marques-Souza, A.C. 1999. Caracteristicas da coleta de pólen de alguns meliponineos da Amazônia Central. Tese de Doutorado, Instituto Nacional de Pesquisas da Amazônia/Fundação Universidade do 
Amazonas. Manaus, Amazonas, 250p.

Neff, J.L.; Simpson, B.B. 1993. Bees, pollination systems and plant diversity. In: La Salle, J.; Gauld, I.D. (Eds.) Hymenoptera and Biodiversity. C.A.B. International, London. p.143-167.

Petanidou, T.; Vokou, D. 1990. Pollination and pollen energetics in mediterranean ecosystems. Amer. J. Bot., 77(8): 986-992.

Petanidou, T. 1992. Measurement of pollen energetic value. In: Dafni, A. (Ed). Pollination ecology - A pratical approach. IRL, Oxford University Press, London. p.131132 .

Pinheiro, M.C.B.; Ormond, W.T.; Lima, H.A.; Correia, M.C.R.; Pimenta, M.L. 1991. Estudo do amido no pólen das angiospermae. Bol. Mus. Nac., N.S., Bot., 86: 1-22.

Pla Dalmau, J.M. 1961. Pollen. Talheres Gráficos. D.C.P., Gerona, 510p.

Ramalho, M. 1990. Foraging by stingless bees of thr genus, Scaptotrigona (Apidae, Meliponinae). J. Apic. Research, 29(2): 61-67.

Real, L.; Rathcke, B.J. 1991. Individual variation in nectar production and its effect on fitness in Kalmia latifolia. Ecology, 72(1): 149-155.

Roubik, D.W.; Buchmann, S. 1984. Nectar selection by Melipona and Apis mellifera (Hymenoptera: Apidae) and the ecology of nectar intake by bee colonies in a tropical forest. Oecologia, 61: 1-10.

Santos, T.C.T. 1991. Dados da obtenção de pólen por operárias de Melipona seminigra merrillae Cock. em Manaus. Dissertação de Mestrado, Instituto Nacional de Pesquisas da Amazônia/ Fundação Universidade do Amazonas. Manaus, Amazonas, 92p.

Silveira, F.A. 1991. Influence of pollen grain volume on the estimation "of the relative importance of its source to bees. Apidologie, 22: 495-502.

Simpson, B.B.; Neff, J.L. 1983. Floral rewards: alternatives to pollen an nectar. Ann. Missouri Bot. Gard., 68: 301-322.
Southwick, E.E.; Loper, G.M.; Sadwick., S.E. 1981. Nectar production, composition, energetics and polinator attractiveness in spring flowers of western New York. Amer, J. Bot. 68: 994-1002.

\section{Aceito para publicação em 01/04/2002}

